\title{
SPATIAL PROCESSING OF SENTINEL IMAGERY FOR MONITORING OF ACACIA FOREST DEGRADATION IN LAKE NAKURU RIPARIAN RESERVE
}

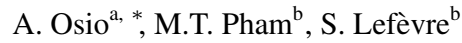 \\ a Technical University of Kenya, Nairobi, Kenya \\ ${ }^{\mathrm{b}}$ Univ. Bretagne Sud, UMR 6074, IRISA, F-56000 Vannes, France
}

KEY WORDS: Attribute Profiles, Haralick Features, Sentinel-1, Sentinel-2, OBIA, Supervised Classification, Vegetation Monitoring

\begin{abstract}
:
Tree degradation in National Parks poses a serious risk to the birds and animals and to a larger extent the general ecosystem. The essence of Forest degradation mapping is to detect the extent of damage on the trees over time, hence providing stakeholders with a basis for forest rehabilitation and intervention. The study proposes a workflow for detection and classification of degrading acacia vegetation along Lake Nakuru riparian reserve. Inspired by previous research on the use of Dual Polarized Sentinel 1 Ground Range Detected (GRD) data for vegetation detection, a set of six Sentinel 1 GRD and Sentinel 2 MSI of corresponding dates (2018-2019) were used. Our study confirms the existing correlation between vegetation indices derived from optical sensors and the backscatter indices from S1 SAR image of the same land cover classes. Factors that were used in validating the results include some comparisons between pixelwise and object-based classification, with a focus on the underlying segmentation and classification algorithms, the polarimetric attributes (VV+VH intensity bands) and the reflectance bands (NIR, SWIR \& GREEN), the Haralick features (GLCM) vs. some geometric attributes (area \& moment of inertia). Classification carried out on the temporal datasets considering geometric attributes and the Random Forest classifier yielded the highest Overall Accuracy (OA) with 94.25\%, and a Kappa coefficient of 0.90.
\end{abstract}

\section{INTRODUCTION}

Wetlands are the most productive ecosystems hosting different types of insects, birds, and large mammals. Lake Nakuru being amongst the Kenya Rift valley Lakes listed amongst wetlands of International Importance hence needs to be preserved. On the shores of Lake Nakuru thrives Acacia Xanthophloea whose occurrence is associated with the high water table. Recently, the lake has been flooding its banks (Onywere et al., 2013) (Fig. 1), thus destroying the yellow barked Acacia whose leaves provide fodder for the Rothschild giraffe and it's pod important feed for the Vervet monkeys in the Park. Since the acacia provides a buffer against siltation in the lake, their degradation could be one amongst other reasons behind the flooding lake.

In order to monitor degrading wetlands, microwave sensors using Radar Technology are appealing since they capture ground information during the day and night independent of changing weather conditions (Woodhouse, 2006), conversely to the optical based sensors. Quantifying the physical characteristics of the backscatter mechanism and relating it to the biophysical properties of vegetation canopies in relation to the radar signals can give leads to the health and structural condition of the riparian vegetation. The capability of backscatter $\sigma^{\circ}$ coefficient correlated to the angle of inclination at the time of capture have been used to give qualitative information about terrain features. Although wetland classification falls under land cover mapping, classes related to riparian reserve and their product utility for various applications still remain uncertain (Krankina et al., 2010). SAR-C polarimetric intensity bands $(\mathrm{VV}+\mathrm{VH})$ have been used in previous studies to map land use and land cover, and further in conjunction with Haralick features (Haralick et al., 1973) to map sea-ice-type (Liu et al., 2015) and in ice water discrimination (Zakhvatkina et al., 2017).

The main purpose of this study is to explore the capability of Sentinel 1 SAR-C polarimetric features in discriminating flooded ri-

\footnotetext{
${ }^{*}$ Corresponding author.
}

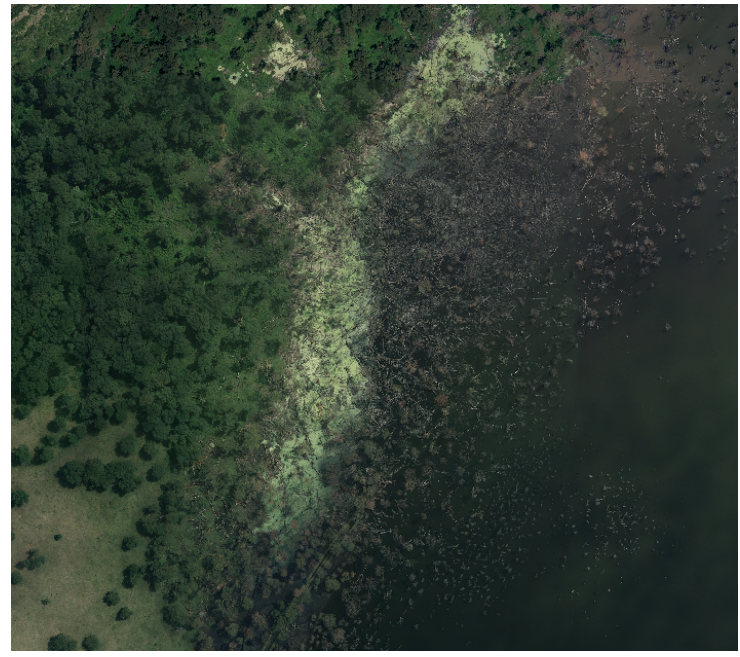

Figure 1: Illustration of the deforestation problem around the Nakuru lake.

parian vegetation. We hypothesized that SAR-C band of Sentinel1 time series stacks analyzed through their Haralick features could be a reliable solution for discriminating flooded vegetation along the degrading Lake Nakuru riparian reserve. Furthermore, following a recent study (Pham et al., 2018a) demonstrating the relevance of attribute profiles w.r.t. Haralick features on optical images, we also hypothesized that these profiles can be used for SAR-C backscatter intensity bands to help discriminating the flooded vegetation classes. Finally, we also hypothesized that the OBIA framework could be successfully applied on temporal stack of Haralick features extracted from Sentinel-1 images in order to increase wetland classification accuracy. 


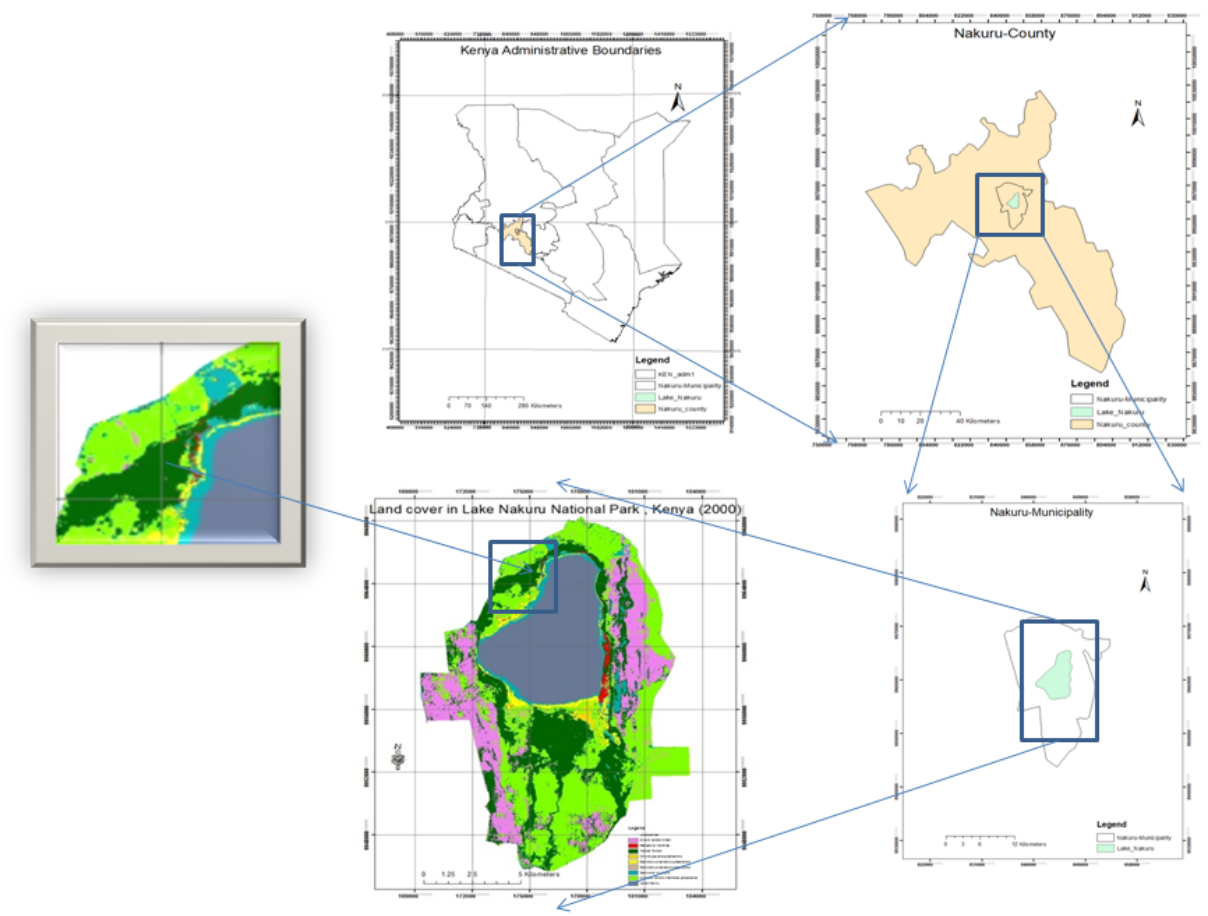

Figure 2: Study Area, located in Nakuru County within Nakuru Municipality $\left(0^{\circ} 18^{\prime} \mathrm{S} \rightarrow 0^{\circ} 27^{\prime} \mathrm{S}, 36^{\circ} 1.5^{\prime} \mathrm{E} \rightarrow 39^{\circ} 9.25^{\prime} \mathrm{E}\right)$, Kenya.

\section{MATERIAL AND METHODS}

\subsection{The study area}

The study area is located in Nakuru County within Nakuru Municipality between $0^{\circ} 18^{\prime} \mathrm{S}$ and $0^{\circ} 27^{\prime} \mathrm{S}$, and $36^{\circ} 1.5^{\prime} \mathrm{E}$ and $39^{\circ} 9.25^{\prime} \mathrm{E}$, within the Kenyan Rift valley (see Fig. 2). The altitude ranges from $1760-2080 \mathrm{~m}$ above sea level. Mean annual rainfall ranges between $876 \mathrm{~mm}$ and $1050 \mathrm{~mm}$ and has an inherent bimodal pattern. The long rains start in March and end in June while the short rains occur between October and December. Mean daily minimum and maximum temperatures fluctuate between $8.2^{\circ} \mathrm{C}$ and $25.6^{\circ} \mathrm{C}$. Rivers that drain their waters into the lake through the park are rivers Lamuriak, Makalia, Naishi, Nderit and Njoro. Classes of vegetation observed in the park were different types of grass species which form the dominant occurrence inside the park. The wetland vegetation includes the yellow barked Acacia Xanthophloea existing along lake's riparian reserve. Most of the vegetation in the park has their peak growing season in the middle of the long wet season (May) and their lowest growing period at the end of February. However, due to the recent effects emanating from climate change dynamics, the dates have been fluctuating.

In another study (Osio et al., 2018), we have confirmed that Lake Nakuru wetland vegetation has been affected by the gradual flooding of the lake in recent times. It revealed that some of the wetland classes such as Acacia xanthophloea forest, chloris gayana grasslands, Cynodon-chloris-Themada grasslands, sporobolus spicatus grasslands, cynodon-niemfluensis-woodedacacia grasslands, sand and mudflats, sedges and marshes had been affected by the flooding lake. NDVI values of the park vegetation had drastically decreased from +0.63 in 2011 to +0.16 in 2016. The difficulty of mapping wetland classes lies with the fact that some of the land cover classes outside Lake Nakuru National Park adapt to the same spectral reflectance signatures as those on the wetland zone along the shores of the lake.

\subsection{Data Pre-processing}

In this study, we consider both SAR and optical images provided by $\mathrm{S} 1$ and $\mathrm{S} 2$ missions respectively, considering the following acquisition dates: 26/05/2018, 25/07/2018, 10/11/2018, $12 / 12 / 2018,02 / 02 / 2019,05 / 05 / 2019$ and 13/08/2019 for $\mathrm{S} 1$; 27/05/2018, 16/07/2018, 23/11/2018, 12/01/2019, 02/05/2019 and 10/08/2019 for S2. All the data were in the same datum and projection i.e. WGS 84 / UTM Zone 36N.

2.2.1 Sentinel-1 Pre-processing Raw $\mathrm{S} 1$ time series data acquired in different seasons (i.e., both rainy and dry seasons) in the years 2018 and 2019 were imported into the ESA open-source software SNAP, with the following pre-processing steps: application of the orbit file on each image, thermal noise removal, radiometric calibration, speckle filtering, terrain correction, ROI clipping with land-sea masking and Haralick feature extraction. Modelling SAR backscatter indices for their use on riparian vegetation requires conversion of $\mathrm{VV}$ and $\mathrm{VH}$ bands into decibel units, i.e. $\sigma_{d b}=10 \log _{10}\left(\sigma^{\circ}\right)$, where $\sigma^{\circ}$ and $\sigma_{d b}$ are respectively the digital number values and the backscattered values (in $\mathrm{dB}$ ).

Extraction of Attribute profiles Among a great number of spatial-spectral feature extraction techniques applied to pixellevel classification of remote sensing data, morphological attribute profiles (APs) have been widely exploited thanks to their capacity to model multilevel spatial information of the image content (Dalla Mura et al., 2010, Pham et al., 2018b). By well preserving significant spatial properties of regions and objects such as contours, shape, etc., APs become effective to characterize the contextual information of the observed scene, hence relevant for a classification purpose. Following the recent suggestion from (Pham et al., 2018a) for optical imagery, we replace the extraction of GLCM textural features with AP extraction for better characterization of structural and textural information within the observed zones from S1 intensity images after the above preprocessing step. The generation of APs from an image can be 
summarized as a four-step process: 1) construct a morphological hierarchy (a.k.a. tree) from the image; 2) compute some relevant attributes (area, moment of inertia, standard deviation, and so on) from each region associated with each node of the tree; 3 ) filter the tree by keeping/removing nodes according to their attribute values; and 4) reconstruct the image from the filtered tree. Steps 3) and 4) can be done for different attributes (with different threshold values) to finally produce a set of filtered images (by stacking them) to form APs.

2.2.2 Sentinel-2 Pre-processing Beyond S1 data, we also rely on Sentinel-2 Multispectral Imagery for the purpose of training polygon extraction (Gordana and Ugur, 2018) to be used in the corresponding S1 classification. The SNAP toolbox is also exploited to pre-process raw S2 data, that were atmospherically corrected independently using Sen2cor Processor, before we apply cloud masking. All bands of interest in this study, i.e. band 3 (green), band 8A (NIR), band 11 (SWIR) and band 12 (SWIR) were resampled at $10 \mathrm{~m}$ spatial resolution using nearest neighbour re-sampling in SNAP.

\subsection{Data Analysis}

Once both S1 and S1 image time series are pre-processed, we conduct a data analysis procedure that makes use of both sources as follows.

2.3.1 Sentinel-1 Data Analysis From the pre-processed S1 products were derived eight bands per date: $\sigma(\mathrm{VV}), \sigma(\mathrm{VH})$, $\sigma(\mathrm{VV})$ mean, $\sigma(\mathrm{VV})$ contrast, $\sigma(\mathrm{VV})$ variance, $\sigma(\mathrm{VH})$ mean, $\sigma(\mathrm{VH})$ contrast and $\sigma(\mathrm{VH})$ variance. Stack averaging was then performed for each band, allowing to project all temporal information into a single, representative image.

We then apply a supervised Random Forest algorithm to classify the wetland classes on the NW part of Lake Nakuru wetland. Due to the dynamism of the lake riparian reserve, training polygons to be used for the classification were derived from S2 data of the corresponding S1 image of the same month (see next subsection). The reference images used for validation come from some high spatial resolution aerial images captured in 2015 on which visual mapping was achieved.

2.3.2 Sentinel-2 Data Analysis For the $\mathrm{S} 2$ images, we extract training polygons by relying on color composition (RGB band combination: 8A,11,12) as suggested by (Gordana and Ugur, 2018). Furthermore, to ease visual assessment, we also derive Normalized Burnt Ratio Index (NBR) and Normalized Difference Water Index (NDWI) values, since the study area is a wetland. These additional bands allow us to get a clear map or image of the study site (see Fig. 3) which can be subsequently used as a reference image for each corresponding classified $\mathrm{S} 1$ image. We recall that NBR and NDWI are computed as follows:

$$
\begin{aligned}
& N B R=\frac{B 8 \mathrm{~A}-B 12}{B 8 \mathrm{~A}+B 12} \\
& N D W I=\frac{B 3-B 8 \mathrm{~A}}{B 3+B 8 \mathrm{~A}}
\end{aligned}
$$

The main reason for using NBR and NDWI indices was to distinguish between the dry-land and the flooded areas. In order to investigate the correlations between $\mathrm{S} 1$ reflectance values $\left(\sigma_{d b}\right)$ in $\mathrm{dB}$ and the NBR reflectance values on the various wetland classes, a correlation analysis was conducted. The crosscorrelation analysis derived from the time series data on Table 1 and results of inter-class correlations measured with Pearson's correlation coefficient are shown on Table 2 where relationships between the backscatter index $\sigma_{d b}(\mathrm{VH})$ from Sentinel-1 and the
NBR reflectance from Sentinel-2 are emphasized. The NBR values for each corresponding date and land-cover class were derived from the SNAP toolbox during image post-processing. Vegetation reflects more in the Near Infrared (NIR) section of the electromagnetic spectrum but less in the Short Wave Infrared (SWIR) region and thus the NBR mostly used for pre-fire and post-fire studies can also be used to measure the health of the trees or vegetation in general. According to previous studies, higher NBR values indicate healthy vegetation while low NBR values indicate degraded one.

\section{RESULTS AND DISCUSSIONS}

Our study exploited S1 and S2 data to characterize the condition of the degrading Acacia Forest along Lake Nakuru National park and further used SAR backscatter coefficient $\sigma_{d b}$ to map the effect of SAR returns on the Acacia xanthophloea spp. To the best of our knowledge, no research studies have used SAR backscatter indices in discriminating the condition of tree degradation along Lake Nakuru riparian reserve. Our research aims at addressing the following knowledge gaps:

1. Identify a robust and reliable method that can be used in tree condition and health detection especially on Acacia Xanthophloea strands along Lake Nakuru riparian reserve.

2. Produce clear and reliable mapping products validated using up-to-date temporal reference maps and machine learning approaches.

3. Explore the capability of $\sigma_{d b}(\mathrm{VV})$ and $\sigma_{d b}(\mathrm{VH})$ backscatter and their Haralick features in detection of the condition of Acacia trees along Lake Nakuru riparian reserve.

4. Explore the capability of the morphological attribute profiles in the discrimination of wetland classes along Lake Nakuru riparian reserve.

In the sequel, we address these questions in some dedicated subsections.

\subsection{Characterization of condition and health of Acacia Xan- thophloea using Sentinel 1 time series datasets}

The NBR values emanating from SITS captured between May, 2018 (0.76) and May, 2019 (0.42) revealed that the yellow barked Acacia have been degrading. To characterize the health of the riparian vegetation, S1 SAR time series with the C-band were used as inspired by previous studies.

Tree condition and health were characterized by generating the spectral signatures and the backscatter indices $\sigma_{d b}(\mathrm{VV})$ and $\sigma_{d b}(\mathrm{VH})$. Figure 5 shows the capability of the backscatter indices for detecting the condition and health of riparian vegetation along the flooding lake. The land-cover backscatter returns from the highest to the lowest were captured as urban, non-degraded forest, degraded forest, degraded submerged forest, and lastly Lake Nakuru. High backscatter response from the urban class was due to double bounce scattering inherent in built up areas. Specular reflectance from Lake Nakuru caused low backscatter response as inspired by previous research (Hess et al., 1995). The backscatter indices $\sigma_{d b}(\mathrm{VV})$ and $\sigma_{d b}(\mathrm{VH})$ had the capability of detecting four classes: degraded submerged forest, degraded forest, non degraded forest and urban. Tree strands consist of crown and the bark. Ground observation revealed massive destruction involving the degrading trees, where some of the trees have dried up and 

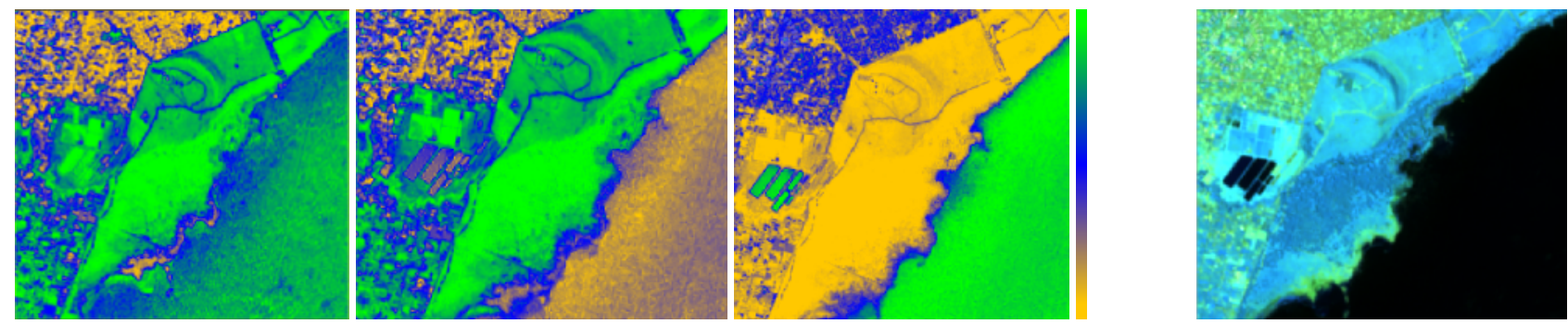

Figure 3: Spectral indices on Sentinel-2 (10/08/2019): Left (from left to right): NBR, NBR-NDWI, NDWI, and color scale. Right: S2 MSI natural image (with spectral bands 12,11 and $8 \mathrm{~A}$ ).

Table 1: Time series backscatter response from Sentinel-1 $\left(\sigma_{d b} \mathrm{VH}\right)$ and spectral values from Sentinel-2 (NBR).

\begin{tabular}{|c||c|c|c|c|c||c|}
\hline Date & S1 Lake & S1 Forest_non_deg & S1 Deg_sub_forest & S1 Deg_forest & S1 Urban & S2 (NBR) \\
\hline \hline July, 2018 & -14 & -15 & -25 & -14 & -13 & 0.69 \\
\hline Nov, 2018 & -12 & -26 & -26 & -22 & -19 & 0.60 \\
\hline Feb, 2019 & 24 & 10 & 6 & 13 & 18 & 0.5 \\
\hline May, 2019 & 24 & 12 & 3 & 13 & 21 & 0.42 \\
\hline Aug, 2019 & -13 & -25 & -27 & -25 & -18 & 0.66 \\
\hline
\end{tabular}

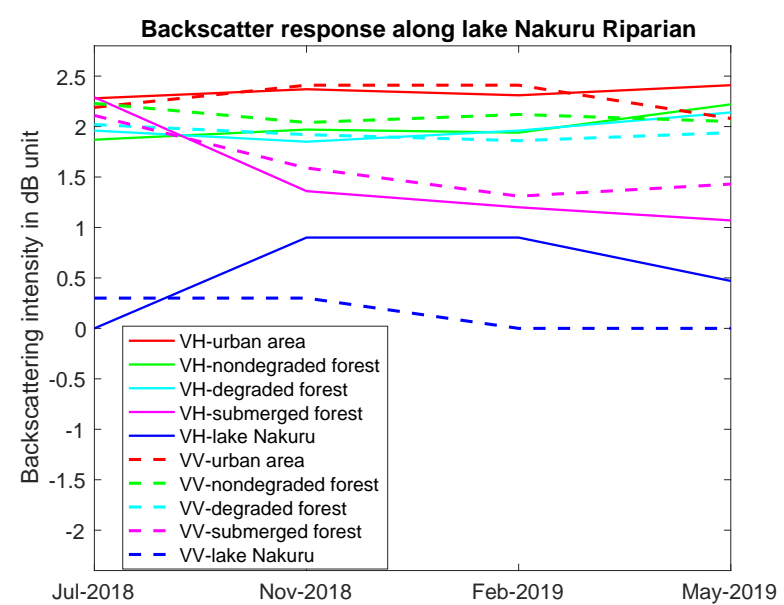

Figure 4: Time series of Sentinel-1 backscatter coefficient $\sigma_{d b}(\mathrm{VV})$ and $\sigma_{d b}(\mathrm{VH})$ on Lake Riparian classes.

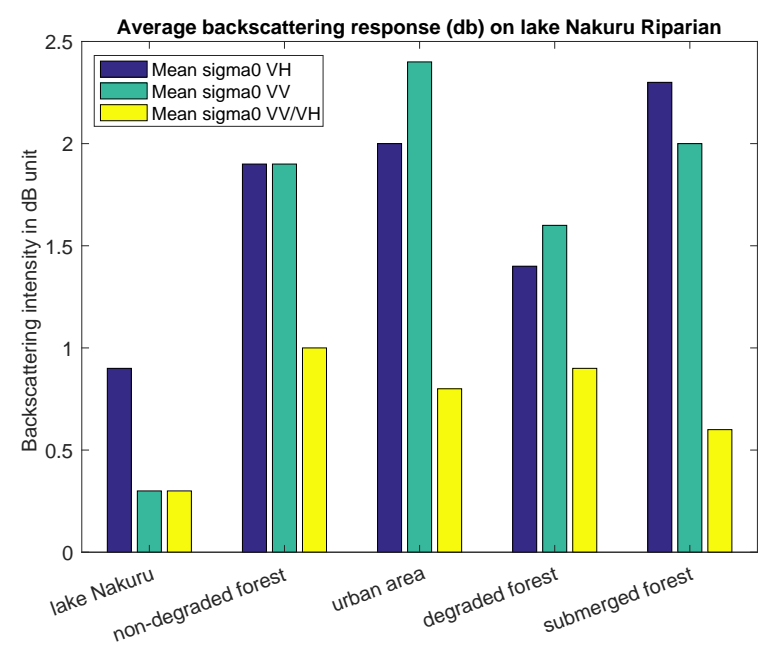

Figure 5: Average backscatter $\sigma_{d b}$ response on Lake Nakuru Riparian classes.

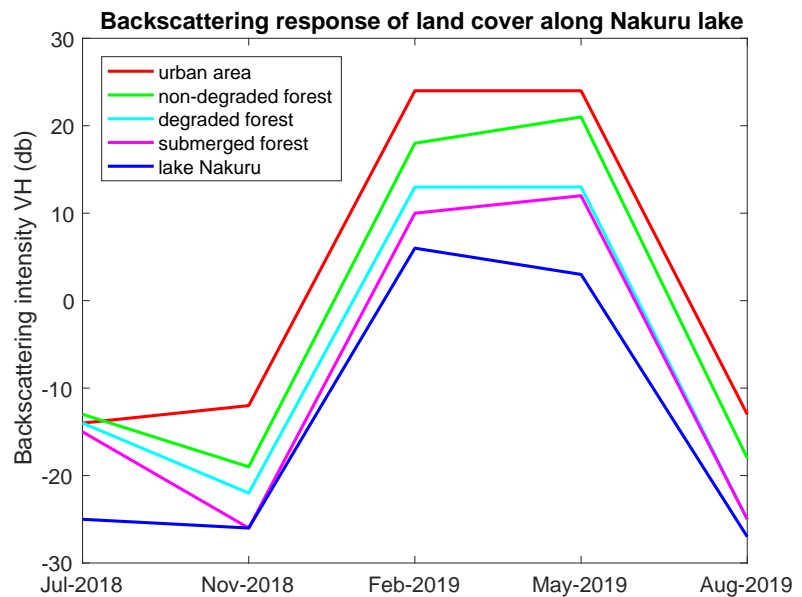

Figure 6: Time series of the backscatter response $(\mathrm{db})$ on riparian land cover classes

standing while others have fallen off the ground. The backscatter response of tree trumps which falls under degraded submerged forest class were captured both in $\sigma_{d b}(\mathrm{VV})$ and $\sigma_{d b}(\mathrm{VH})$ polarization modes. According to previous studies (Richards et al., 1987), the backscatter response emanating from the water surface undergoes double bounce phenomena to and from the flooded vegetation which in this case consists of dead tree trunks in water. Finally the backscatter signals are relayed back to the antennae of the SAR instrument. The degraded forest and urban classes were captured in $\mathrm{VV}$ polarization mode, non-degraded forest was best captured in $\mathrm{VH}$ polarization mode since healthy tree strands experience volume scattering on the crown and double bounce from their bark. It is known that the backscatter response can be captured from vegetation only in the early phenological stages. Acacia xanthophloea strands on the riparian reserve are mature trees that have been in existence for many years. The backscatter response patterns as observed in (Kasischke et al., 2009) were evident in this study where the increase in backscatter response signals was observed at growing levels for the open water (OW), dry land (DL) and flooded vegetation (FV). However, according to (White et al., 2015), the patterns are influenced by several factors such as radar wavelength, polarization, angle of incidence, open water surface roughness, topography, vegetation type and 
Table 2: Synergy between Sentinel-1 $\left(\sigma_{d b} \mathrm{VH}\right)$ and Sentinel-2 (NBR).

\begin{tabular}{|c||c|c|c|c|c||c|}
\hline Correlations & S1 Lake & S1 Forest_non_deg & S1 Deg_sub_forest & S1 Deg_forest & S1 Urban & S2(NBR) \\
\hline \hline S1 Lake & 1.0 & & & & & \\
\hline S1 Forest_non_deg & 0.964 & 1.0 & & & & \\
\hline S1 Deg_sub_forest & 0.996 & 0.975 & 1.0 & & & \\
\hline S1 Deg_forest & 0.971 & 0.996 & 0.983 & 1.0 & & \\
\hline S1 Urban & 0.988 & 0.993 & 0.990 & 0.991 & 1.0 & \\
\hline \hline S2(NBR) & -0.933 & -0.854 & -0.901 & -0.868 & -0.902 & 1.0 \\
\hline
\end{tabular}

phenology.

\subsection{Production of reliable mapping products validated us- ing up-to-date temporal reference maps and machine learning approaches}

In this section, the Sentinel-1 capability in the classification of riparian vegetation classes was tested. In order to determine the effect of Haralick features on classification accuracy, the inclusion of the Haralick features in VV and VH SAR intensity bands was embraced. Object-based and pixelwise supervised classification were carried out on the different datasets as follows:

1. The test dataset was a Very High Resolution imagery (VHR) at 10-cm spatial resolution captured in the year 2015 when the lake was already flooding its banks (Figure 1). Sample based classification was carried out to produce four classes namely degraded acacia, degraded submerged acacia, non degraded acacia, and Lake Nakuru. Multi-resolution segmentation was appended, setting scale parameters at scale 73 , shape 0.6 and compactness 0.8 . In order to promote class separation, four Haralick features were appended namely GLCM-Mean, GLCM-Contrast, GLCM-Homogeneity and GLCM-Correlation. The user-defined test dataset was processed using Random forest yielding 99.7\%, Multi-Layer perceptron $91.6 \%$, and Binary Support Vector (SMO) at $75 \%$ accuracy respectively.

2. The second test dataset was made of 8 pre-processed $\mathrm{S} 1$ Ground Range detected (GRD) imagery and their corresponding Haralick features captured between May 2018 and August 2019. The images of different temporal dates were combined to form a single stack image consisting of 32 master and slave images. Stack averaging was performed giving rise to an image with eight features. Superpixel segmentation (SLICO) with minimum region size of 25 was used. For further class refinement, a spectral difference segmentation at scale 10 was applied in order to create distinct boundaries between classes. Classification accuracy yielded the following results: Random Forest $82.3 \%$, Multilayer perceptron $80.3 \%$, and binary Support vector (SMO) 76.6\% respectively.

3. Pixel-wise supervised classification was carried out on three datasets which consist of Sentinel-1 Level-1 Ground range (GRD) detected of VV and VH intensity bands. The Haralick features included in the classification were GLCMmean, GLCM-contrast and GLCM-variance hence giving rise to a total of 8 bands per each image. Random forest (500 trees) classification was used with 5000 samples used for training and 500 samples for validation. This procedure was carried out in ESA SNAP environment. The Overall Accuracy (OA) achieved from the classified image of the study area captured on July 2018 was 96\%, November 2018 $94 \%$, and May $201995 \%$ respectively.
4. Supervised object-based classification on lake riparian classes was done using a different approach. Since the riparian classes were dynamic due to the persistent flooding, S2 images of the corresponding S1 SAR images were used in collection of training sets as demonstrated from recent studies by (Gordana and Ugur, 2018) while the in-situ dataset was a VHR aerial imagery of $10 \mathrm{~cm}$ ground resolution (Figure 1). Random forest classification appended on three S1 datasets captured in February 2019, May 2019 and August 2019 yielded overall classification accuracy of $89.2 \%, 87.9 \%$, and $94 \%$ respectively (Table 3 ).

3.3 Exploring the capability of $\sigma \mathrm{VV}+\mathrm{VH}$ polarimetric indices and their Haralick features in the detection of condition and health of Acacia xanthophloea along Lake Nakuru riparian reserve

S1 polarimetric features are suitable for the detection of riparian vegetation classes along Lake Nakuru riparian reserve. This can be observed in Table 3 with Random Forest classification results of $87.7 \%, 84.93 \%$ and $92.45 \%$ for the 3 dates. Furthermore, incorporating the Haralick features improved by $1 \%$ to $3 \%$ the classification result. This improvement is consistent for all images, leading even to a $93.95 \%$ overall accuracy.

3.4 Exploring the capability of Attribute Profiles in the discrimination of wetland classes along Lake Nakuru riparian reserve

The final objective of this study was to investigate the capability of engaging attribute profiles derived from the 8 bands coming from Sentinel-1 SAR imagery and compare the results with different datasets and different attributes. The eight bands included $\sigma_{d b}(\mathrm{VV}), \sigma_{d b}(\mathrm{VH}), \sigma_{d b}(\mathrm{VV})$ GLCM-mean, $\sigma_{d b}(\mathrm{VV})$ GLCM-variance, $\sigma_{d b}(\mathrm{VV})$ GLCM-contrast, $\sigma_{d b}(\mathrm{VH})$ GLCMmean, $\sigma_{d b}(\mathrm{VH})$ GLCM-variance, and $\sigma_{d b}(\mathrm{VH})$ GLCM-contrast. Attribute profiles were derived from these 8 bands and used in the classification of Lake Nakuru riparian reserve, mainly on the North Western part where flooding has caused tree degradation. Visual comparison between Haralick features and AP is given in Figure 8. The results of the classification using Random Forest algorithm are shown in Table 3. The overall accuracy (OA) reached $90.88 \%, 90.48 \%$ and $94.25 \%$ for the 3 dates. It was obtained using the self-dual APs with two geometric attributes including area and moment of inertia. Manual setting of attribute thresholds has been done based on the spatial resolution of Sentinel-1 images. Four thresholds were set for each attribute: $\lambda_{a}=\{100,500,1000,5000\}$ for area and $\lambda_{i}=\{0.2,0.3,0.4,0.5\}$ for moment of inertia, following the parameter setting guide provided in (Pham et al., 2017, Pham et al., 2018b). The generation of APs was performed on each band of $V V$ and $V H$, then all profiles were stacked to form the AP feature vector and perform Random Forest classification. 
Table 3: Comparative results.

\begin{tabular}{|c|ccc|ccc|ccc|}
\hline \multirow{2}{*}{ Approach } & \multicolumn{3}{|c|}{$\mathbf{0 2 / 0 2 / 2 0 1 9}$} & \multicolumn{3}{c|}{$\mathbf{0 5 / 0 5 / 2 0 1 9}$} & \multicolumn{3}{c|}{$\mathbf{1 3 / 0 8 / 2 0 1 9}$} \\
\cline { 2 - 9 } & OA & AA & kappa & OA & AA & kappa & OA & AA & kappa \\
\hline Intensity & $87.76 \%$ & $72.77 \%$ & 0.7917 & $84.93 \%$ & $69.17 \%$ & 0.7918 & $92.45 \%$ & $76.97 \%$ & 0.8699 \\
GLCM & $89.22 \%$ & $75.42 \%$ & 0.8159 & $87.89 \%$ & $73.58 \%$ & 0.7958 & $93.95 \%$ & $82.55 \%$ & 0.8962 \\
AP & $90.88 \%$ & $78.18 \%$ & 0.8435 & $90.48 \%$ & $76.98 \%$ & 0.8362 & $94.25 \%$ & $85.40 \%$ & 0.9019 \\
\hline
\end{tabular}

\section{DISCUSSIONS}

The study demonstrates how Sentinel-1 backscatter coefficient $\sigma_{d b}(\mathrm{VV})$ and $\sigma_{d b}(\mathrm{VH})$ can be used to characterize wetland degradation. While it is well-known that SAR polarimetric analysis of electromagnetic waves enables discrimination of different scattering mechanisms emanating from the ground, we have found $\sigma_{d b}(\mathrm{VV})$ and $\sigma_{d b}(\mathrm{VH})$ to be less sensitive to the scattering mechanisms $(+0.9 \mathrm{db})$ emanating from Lake Nakuru riparian mainly because Acacia xanthophloea are mature trees that have been in existence on the riparian reserve for a long time. It was worth noting that the SAR C-band response in the $\sigma_{d b}(\mathrm{VH})$ was sensitive to moisture content in the leaves of the healthy trees as captured in the peak season (June, 2019) when average rainfall distribution was at its peak in the study area (Figure 5) as captured in previous research (Koppe et al., 2013). The backscatter $\sigma_{d b}(\mathrm{VH})$ had the lowest average response on class Lake Nakuru $(+0.3)$ in $\sigma_{d b}(\mathrm{VV})$. This was confirmed by existing studies (Muster et al., 2013) that report small lakes reduce the backscatter response due to their smooth surfaces. The degraded submerged forest had equal average backscatter response $(+1.9 \mathrm{db})$ in $\sigma_{d b}(\mathrm{VV})$ and $\sigma_{d b}(\mathrm{VH})$, as shown on Figure 6. This part of the wetland consists of dead and semi-dead biomass lying on the mudflats on the riparian reserve. SAR-C polarimetric electromagnetic waves have short wavelengths i.e. $3.75 \mathrm{~cm}$ to $7.5 \mathrm{~cm}$ and hence are unlikely to penetrate through the biomass and only represent a few centimetres of signals emanating from the surface of the degrading biomass thus excluding the response from the wet soils beneath the mass. Another aspect which could contribute to the higher average backscatter was the presence of biomass in open water (Lake Nakuru), meaning that the response was a mixture of specular reflectance from the open water and double bounce from the biomass in water (degraded-submerged forest) as confirmed by previous studies (Kasischke et al., 2009). From the class forest degraded, the highest average backscatter response (+1.6) was achieved by $\sigma_{d b}(\mathrm{VV})$. Most of the biomass was in class degraded submerged, hence the difference in backscatter response between the class degraded submerged and forest degraded. This report corroborates previous studies stating that low biomass presence on wetlands caused low backscatter response while higher volumes of biomass caused higher backscatter response. Forest non-degraded forest class which consisted of healthy acacia xanthophloea had the highest average backscatter response $\sigma_{d b}(\mathrm{VH})$ of value $+2.3 \mathrm{db}$ which was higher than the previous classes i.e. forest degraded and degraded submerged hence confirming reports that the dynamic range of the cross-polarized VH component renders it suitable for measuring the backscatter mechanisms of vegetation. Due to the short wavelength inherent in Sentinel1 SAR C $(3.75-7.5 \mathrm{~cm})$, volume scattering occurs on the tree crowns without penetrating the under storey vegetation (Mcnairn and Brisco, 2004).

The highest mean backscatter $\sigma_{d b}(\mathrm{VH})(+2.4 \mathrm{db})$ emanated from the urban class that has double bounce response due to the buildings in the neighboring Nakuru Town. Limitations on the backscatter response can be pointed out on the fact that the values were obtained from user-defined polygons from each class, meaning that the values vary across different sites on each class. Future research should engage collection of backscatter values across each class. However, accessibility to different parts of the affected areas is difficult and thus in-situ data from a Very High Resolution aerial imagery at $10 \mathrm{~cm}$ (Figure 1) ground distant resolution was used. The baseline aerial image at GRD of $10 \mathrm{~cm}$ was used as input of a multiresolution segmentation with subsequent classification with Random Forest, yielding 99.7\% accuracy. This was due to the the fact that VHR imageries have distinct class boundaries. The averaged stack of 32 master and slave imageries yielded $82.3 \%$ overall accuracy. These results could have been influenced by the number of training instances, as well as the number of trees used in the parameterization; 100 trees yielded $100 \%$ overall accuracy on the dataset and hence the number of trees that was set was 30 with the main aim of dealing with overestimation issues. The effect of SAR-C polarimetric intensity bands on classification accuracy was tested. It was worth noting that pixelwise Random Forest classification of Sentinel 1 SAR-C dataset considering $\mathrm{VV}, \mathrm{VH}$, and ratio yielded a high overall accuracy of $92.45 \%$ on the August 2019 image of the study area. Stacking Haralick features on the intensity bands namely GLCM-mean, GLCM-contrast and GLCM-variance improved the classification on the same image by $1.5 \%$ meaning that engaging the GLCM-Haralick feature bands contributed slightly to the improvement of classification accuracy. A further slight improvement of classification accuracy at $1.8 \%$ was noted when Attribute profiles were extracted from the Sentinel 1 bands. The highest commission error was noted with class degraded forest considering both the Haralick and attribute profile based classification. This could have been due to the fact that the degraded forest class lies in the middle of classes degraded submerged forest and the non degraded forest, thus causing confusion between class boundaries. Pixel-wise Random Forest on the single date ( 8 bands, VH, VV and their respective 3 Haralick features) yielded higher $94.0 \%$ overall accuracy. Attribute profile based classification by Random Forest working on 62,067 instances and 200 trees yielded $94.25 \%$ overall accuracy.

\section{CONCLUSION}

This study demonstrates how Sentinel-1 Synthetic Aperture Radar (SAR-C) imagery, Ground Range Detected (GRD) with a wavelength of $3.75-7.5 \mathrm{~cm}$ can be incorporated with Haralick features to discriminate wetland classes along the North Western part of Lake Nakuru riparian reserve. Identifying the various classes and confirming their different backscatter response across different polarimetry opens up new perspectives on their response to vegetation health and structures. The Sentinel- 2 datasets corresponding to the Sentinel-1 datasets were helpful in providing training polygons used for the Haralick and Attribute Profilebased feature extraction and classification, hence avoiding errors due to the dynamism of the flooding Lake over time. The highest classification accuracy received from the different datasets came from the attribute profiles at $94.25 \%$. However, there was no significant difference between the Haralick features (93.95\%) and the outcome of the Attribute profiles. Haralick features embedded on the Synthetic Aperture Radar SAR-C bands could have some limitations due to the short wavelength associated with the electromagnetic spectrum (EMS). Future research should explore the capability of radar imagery with longer wavelengths such 

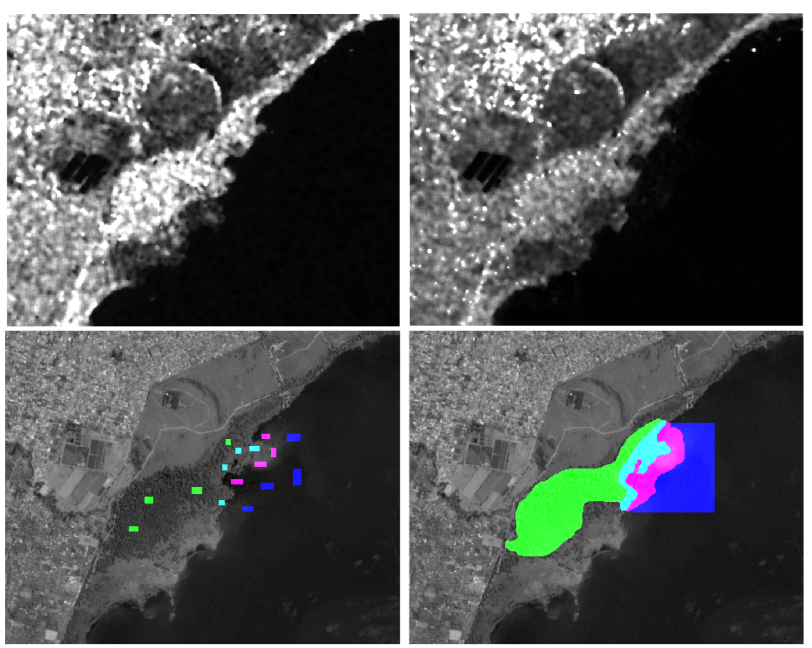

Figure 7: Sentinel-1 VV and VH images (13/08/2019) on the studied area (filtered by $7 \times 7$ Lee filter) together with the train and validation sets (shown on the corresponding Sentinel-2 image). Color codes: Green: healthy forest; Cyan: damaged forest; Magenta: submerged trees; Blue: water.

as the L-band to collect the backscatter response values from the wetland classes. Application of OBIA on Attribute profiles should also be tested in future. Decomposition of quad-polarized Synthetic Aperture Radar polarimetric bands could give leads to structure of the biomass along Lake Nakuru riparian. Given the heterogeneous nature of the study area in comparison with the level of accuracy achieved in this study, we can conclude that the procedures and attributes used in this study could be also used elsewhere where the landscapes are similar.

\section{ACKNOWLEDGEMENTS}

The authors acknowledge: Kenya National Research Fund (K$\mathrm{NRF}$ ) and Campus France through Pamoja-PHC programme; European Space Agency for the provision of Sentinel-1 and Sentinel-2 products; Trimble Inc. Germany for the provision of eCognition Developer Software Licence.

\section{REFERENCES}

Dalla Mura, M., Benediktsson, J. A., Waske, B. and Bruzzone, L., 2010. Morphological attribute profiles for the analysis of very high resolution images. IEEE Transactions on Geoscience and Remote Sensing 48(10), pp. 3747-3762.

Gordana, K. and Ugur, A., 2018. Sentinel-1 and sentinel-2 data fusion for wetland mapping in belgami, turkey. In: ISPRS TC III Mid-Term symposium, The International Archives of Photogrammetry, Remote Sensing and Spatial Information, Vol. 152.

Haralick, R., Shanmugam, K. and Dinstein, I., 1973. Textural features for image classification. IEEE Trans. Syst. Man Cybern. 3(6), pp. 610-621.

Hess, L., Melack, J., Filoso, S. and Wang, Y., 1995. Delineation of innudated area and vegetation along the amazon floodplain with sar-c aperture radar. IEEE Transactions on Geoscience and Remote Sensing 33(4), pp. 896-404.

Kasischke, E., Bourgeau-Chavez, L., Rober, A., Wyatt, K., Waddington, J. and Turetsky, M., 2009. Effects of soil moisture and water depth on ers sar imagery. Remote Sensing of Environment 88(4), pp. 423-441.
Koppe, W., Gynp, M., Hutt, C., Yao, Y., Miao, Y., ChenChen, X. and Bareth, G., 2013. Rice monitoring with multi-temporal and dual polarimetric terrasar-x data. International Journal of Applied Earth Observation and Geoinformation 21, pp. 568-576.

Krankina, O. N., Pflugmacher, D., Hayes, D. J., McGuire, A. D., Hansen, M. C., Häme, T., Elsakov, V. and Nelson, P., 2010. Vegetation cover in the eurasian arctic: Distribution, monitoring, and role in carbon cycling. In: Eurasian arctic land cover and land use in a changing climate, Springer, pp. 79-108.

Liu, H., Guo, H. and Zhang, L., 2015. Svm-based sea ice classification using textural features and concentration from radarsat-2 dual-pol scansar data. IEEE J. Sel. Top. Appl. Earth Observ. Remote Sens.

Mcnairn, H. and Brisco, B., 2004. The application of c-band polarimetric sar for agriculture: a review. Canadian Journal of Remote Sensing 30, pp. 525-542.

Muster, S., Heim, B., Abnizova, A. and J., B., 2013. Water body distributions across scales: A remote sensing based comparison of three arctic tundra wetlands. Remote Sensing 5(4), pp. 1498-1523 .

Onywere, S., Shisanya, C., Obando, J., Ndubi, A., Masiga, D., Irura, Z., Mariita, N. and Maragia, H., 2013. Geospatial extent of 2011-2013 flooding from the east african rift valley in kenya and its implications on the ecosystem. In: Kenya Soda Lakes Workshop.

Osio, A., Lefèvre, S., Ogao, P. and Ayugi, S., 2018. OBIA-based monitoring of riparian vegetation applied to the identification of degraded Acacia Xanthophloea along Lake Nakuru, Kenya. In: GEOBIA 2018 - From pixels to ecosystems and global sustainability, pp. 18-22.

Pham, M., Lefèvre, S. and Merciol, F., 2018a. Attribute profiles on derived textural features for highly-textured optical image classification. IEEE Geoscience and Remote Sensing Letters 15(7), pp. 1125-1129.

Pham, M., Lefèvre, S., Aptoula, E. and Bruzzone, L., 2018b. Recent developments from attribute profiles for remote sensing image classification. In: International Conference on Pattern Recognition and Artificial Intelligence (ICPRAI).

Pham, M.-T., Lefèvre, S. and Aptoula, E., 2017. Local featurebased attribute profiles for optical remote sensing image classification. IEEE Transactions on Geoscience and Remote Sensing 56(2), pp. 1199-1212.

Richards, J., Woodgate, P. and Skidmore, K., 1987. An explanation of enhanced radar backscattering from flooded forests. International Journal of Remote Sensing 8(7), pp. 1093-1100.

White, L., Brisco, B., Dabbor, M. and Schmitt, A., 2015. A collection of sar methodologies for monitoring wetlands. Remote Sensing 7(6), pp. 7615-7645.

Woodhouse, I., 2006. Introduction to microwave remote sensing. Boca Raton: Taylor \& Francis.

Zakhvatkina, N., Korosov, A., Muckenhube, r. S., Sandven, S. and Babiker, M., 2017. Operational algorithm for icewater classification on dual-polarized radarsat-2 images. Cryosphere 11, pp. 33-46. 

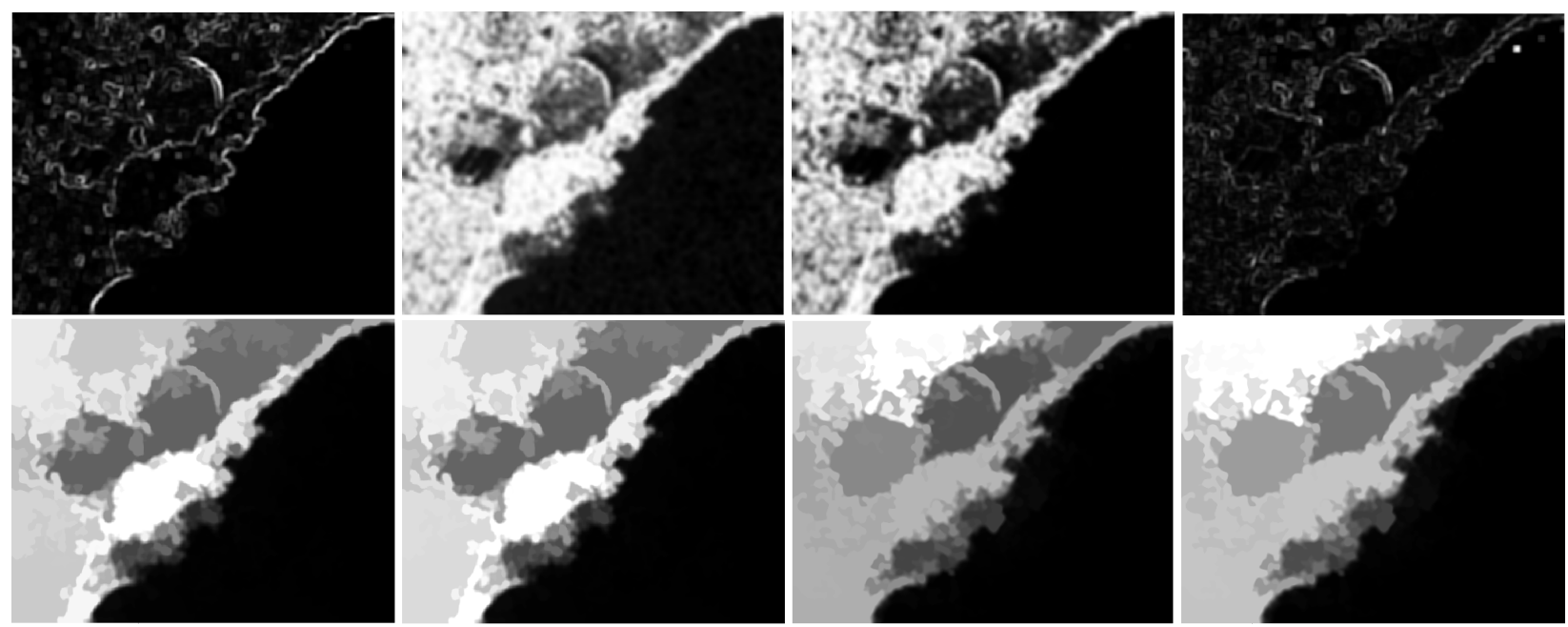

Figure 8: Illustration of Haralick textural features (top line) produced by GLCM method and multilevel AP features (bottom line). 